\title{
The Anopheles Innate Immune System in the Defense against Malaria Infection
}

\author{
April M. Clayton Yuemei Dong George Dimopoulos \\ W. Harry Feinstone Department of Molecular Microbiology and Immunology, Bloomberg School of Public Health, \\ Johns Hopkins University, Baltimore, Md., USA
}

\begin{abstract}
Key Words
Anopheles · Innate immunity · Plasmodium · Malaria · Mosquito
\end{abstract}

\begin{abstract}
The multifaceted innate immune system of insects is capable of fighting infection by a variety of pathogens including those causing human malaria. Malaria transmission by the Anopheles mosquito depends on the Plasmodium parasite's successful completion of its lifecycle in the insect vector, a process that involves interactions with several tissues and cell types as well as with the mosquito's innate immune system. This review will discuss our current understanding of the Anopheles mosquito's innate immune responses against the malaria parasite Plasmodium and the influence of the insect's intestinal microbiota on parasite infection.
\end{abstract}

Copyright $\odot 2013$ S. Karger AG, Basel

\section{Introduction}

Malaria, caused by the Plasmodium parasite, affects approximately 3 billion people worldwide each year. The major vector for Plasmodium falciparum in sub-Saharan Africa is the female Anopheles gambiae mosquito. Given the lack of an effective vaccine against Plasmodium and the increased resistance of this parasite to the current arsenal of drugs and of Anopheles mosquitoes to insecti- cides, the development of novel control strategies is crucial to reducing malaria transmission [1]. Studies exploring the mosquito's innate immune defense against Plasmodium may contribute towards the development of such preventive and control strategies. In this review, we will discuss recent findings from studies investigating anti-Plasmodium defenses in the mosquito, with a specific focus on those involved in parasite elimination in the midgut.

\section{Plasmodium Infection of the Anopheles Mosquito}

Plasmodium transmission requires that the parasite complete an intricate replicative cycle in the mosquito that involves transitions through several developmental stages and interactions with the mosquito's midgut and salivary gland tissues as well as the hemocoel. This journey takes approximately 2-3 weeks (the time varies for different Plasmodium species and strains) and begins when the female mosquito ingests a blood meal infected with Plasmodium gametocytes. The male and female gametocytes develop into male microgametes and female macrogametes, respectively, in the midgut lumen. Fertilization of the gametes results in the formation of zygotes. The zygotes then transform into motile ookinetes that invade and migrate across the midgut epithelium, roughly 18-36 $\mathrm{h}$ after the ingestion of an infected blood meal. The route of ookinete invasion across the midgut epithelium

\section{KARGER}

E-Mail karger@karger.com

www.karger.com/jin
(C) 2013 S. Karger AG, Basel

$1662-811 \mathrm{X} / 13 / 0062-0169 \$ 38.00 / 0$
Dr. George Dimopoulos

W. Harry Feinstone Department of Molecular Microbiology and Immunology Bloomberg School of Public Health, Johns Hopkins University 615 North Wolfe Street, Baltimore, MD 21205 (USA)

E-Mail gdimopou@jhsph.edu 
as well as the cellular responses of the midgut epithelium to ookinete invasion are still topics of controversy despite numerous studies involving diverse Plasmodium-mosquito combinations [2-4]. However, these responses generally involve apoptosis and an extrusion of ookinete-invaded midgut epithelial cells into the midgut lumen [512]. Once the diploid ookinete has reached the basal side of the midgut epithelium, it transforms into an oocyst and undergoes several rounds of replication by means of sporogony. Approximately 10-12 days after the blood meal, each oocyst contains thousands of haploid sporozoites, which are then released into the mosquito hemocoel at about 14 days after the blood meal and migrate through the mosquito hemolymph in order to invade the salivary glands. During the next blood meal, these Plasmodium sporozoites are injected with the saliva into the human (or another vertebrate) host, thereby completing the sexual cycle of Plasmodium within the mosquito vector [13, $14]$.

\section{Mosquito Immune Signaling Pathways in the Defense against Plasmodium}

In order to continue its cycle of transmission and eventual infection of the human host, the malaria parasite engages in a series of complex interactions with the mosquito vector. Parasite numbers are limited by several major bottlenecks that occur in the mosquito such as when the ookinete traverses the midgut epithelium prior to the development of the oocysts on the basal side and during the migration of sporozoites to the salivary glands [1519]. The mosquito's innate immune system has been shown to play a key role in killing parasites and thereby affecting parasite development $[20,21]$. The two major arms of the insect innate immune response are: (1) a humoral response involving, for example, a complementlike system and the transcriptional upregulation of small cationic antimicrobial peptides (AMPs) and other immune effectors and (2) a cell-mediated response that includes phagocytosis and/or melanization. Other defenses include oxidative and nitric oxide (NO)-mediated killing mechanisms.

As earlier mentioned, the innate immune system of Anopheles, the mosquito's main line of defense against parasites, fungi, bacteria and viruses, is engaged at multiple stages of Plasmodium infection [13, 22-24]. Three major signaling pathways contribute to anti-Plasmodium defense: the Toll, the immune deficiency (Imd) and the Janus kinase-signal transducers and activators of tran- scription (JAK-STAT) pathways (fig. 1). Considerable insight into these innate immune pathways has been gathered from studies conducted in Drosophila [24, 25].

\section{The Toll and Imd Pathways}

The mosquito's anti-Plasmodium and antibacterial defenses are largely controlled by the Toll and Imd NF- $\mathrm{BB}$ immune signaling pathways (fig. 1). The Toll pathway is primarily elicited by Gram-positive (G+) bacteria, fungi and Plasmodium. Studies have also implicated this pathway in the defense against viruses [26]. The Imd pathway is elicited by Gram-negative (G-) and $\mathrm{G}+$ bacteria and Plasmodium [22, 27].

Infection-responsive activation of the Toll and Imd pathways via the recognition of PAMPs (pathogen-associated molecular patterns) ultimately leads to the nuclear translocation of the NF- $\kappa \mathrm{B}$ transcription factors Rell and Rel2, respectively (fig. 1). These transcription factors are negatively regulated in the cytoplasm by Cactus and Caspar, respectively. Activation of the Toll and Imd pathways allows the Rel factors to enter the nucleus and transcriptionally activate immune effector genes such as AMPs and other factors. The four main classes of AMPs are defensins, cecropins, attacin and gambicin. These AMPs act against G- and G+ bacteria, yeast, fungi and Plasmodium. Actually, gambicin was among the first anti-Plasmodium factors identified [28]. Additionally, it has been demonstrated that both the Rel1 and Rel2 transcription factors can induce the expression of the AMP genes Cecropin 1, Defensin 1 and Gambicin 1 [29].

The Imd pathway-controlled transcription factor Rel2 gene produces a full-length form (Rel2-F) that includes the carboxyl-terminal ankyrin, ANK, and death domains as well as a shorter form (Rel2-S) lacking such domains due to alternative splicing. The Rel2-S form is constitutively translocated to the nucleus, where it regulates the transcription of immune genes [27] (fig. 1).

While the Toll pathway has been shown to be more effective in the defense against the rodent $P$. berghei parasite, the Imd pathway has emerged as the most effective pathway in the defense against the human malaria parasite $P$. falciparum [30-33]. Specifically, activation of the Imd pathway by the gene silencing, via RNAi, of Caspar (a suppressor of the Imd pathway) results in an Imd pathway-mediated immune defense that confers almost complete refractoriness to $P$. falciparum in three major Anopheles malaria vector species: An. gambiae, An. stephensi and $A n$. albimanus. In contrast, activation of the Toll pathway by the silencing of Cactus (a suppressor of the Toll pathway) results in a significantly greater resistance 


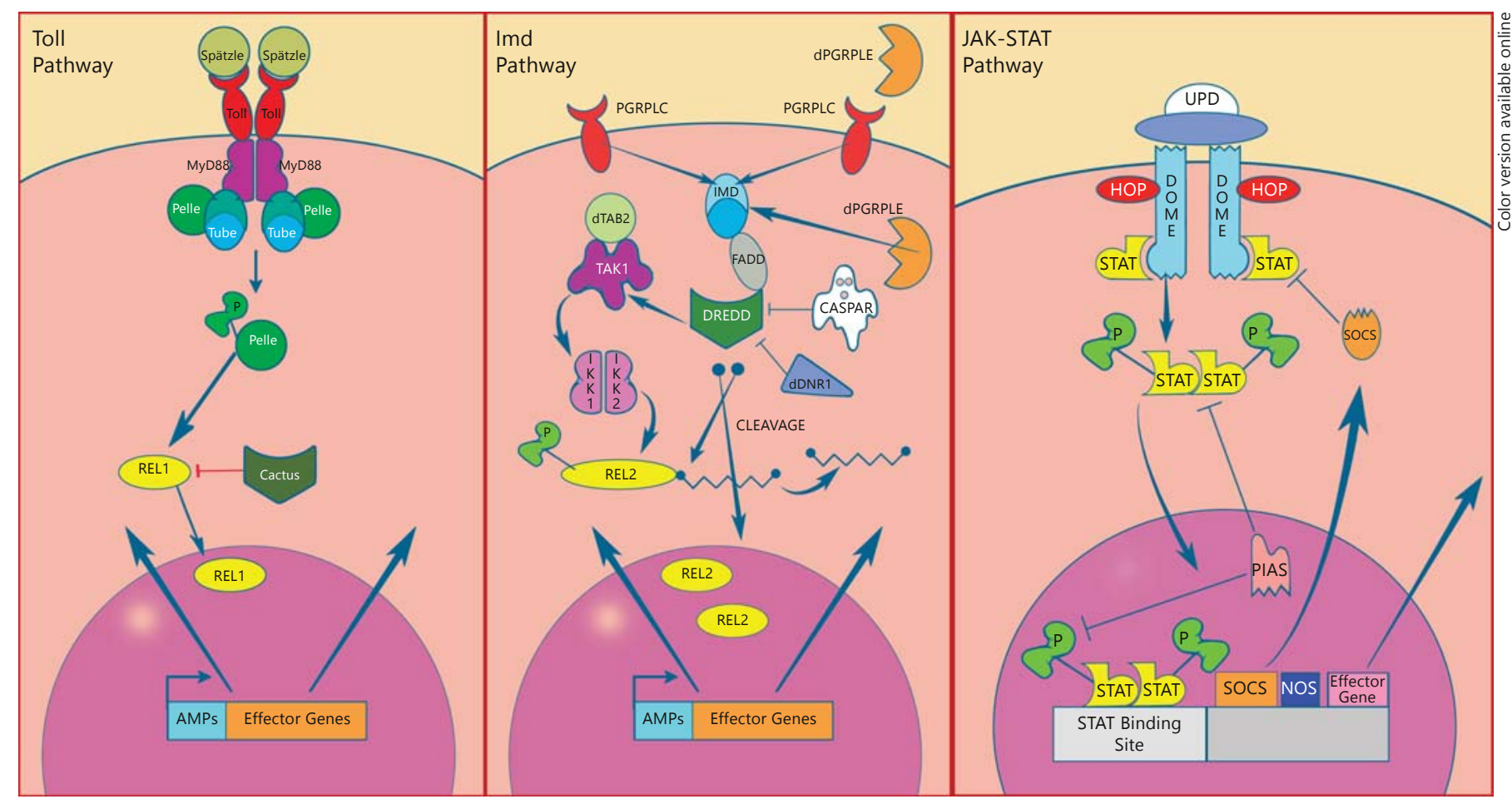

Fig. 1. The Toll, Imd and JAK-STAT immune signaling pathways. Upon the recognition of bacteria or Plasmodium, the Toll pathway is stimulated by the binding of the ligand Spätzle with the Toll transmembrane receptor. This triggers a series of molecular events that culminate in the activation and translocation of Rell into the nucleus, upregulating the transcription of immune genes that are responsible for microbial killing. Numerous studies conducted in Anopheles have highlighted the Imd pathway as the most efficient immune pathway in the defense against the human malaria parasite, $P$. falciparum. The Imd pathway is stimulated when the trans-

to infection with the rodent malaria parasite $P$. berghei [31]. These two Plasmodium species elicit diverse innate immune responses at the gene transcript level [34]. A diverse repertoire of anti-Plasmodium immune effectors regulated by the Imd pathway, including APL1, TEP1, LRRD7 (APL2), FBN9 and LRIM1 have been identified and studied with regard to their antiparasitic action and will be discussed later [27, 33-39]. It has also demonstrated and characterized the potency of the Imd pathway in anti-Plasmodium defense through the use of genetically modified immune-enhanced Anopheles mosquitoes that express blood meal-inducible Rel 2 in both the midgut and fat body tissues [40]. The transient activation of this transgene resulted in almost complete resistance to the human malaria parasite at a negligible fitness cost, prompting further investigation of this system as an innovative malaria control strategy [40]. membrane PGRP-LC receptor recognizes bacteria or Plasmodium. This leads to a signaling cascade that will result in the cleavage of Rel2-F and the translocation of active Rel2-S into the nucleus, upregulating the transcription of immune genes. The JAK-STAT immune signaling pathway has been implicated in antibacterial, antiviral and antiplasmodial defense in mosquitoes. The JAK-STAT pathway is initiated by the binding of the cytokine ligand UPD to the transmembrane receptor DOME. This then leads to the eventual nuclear translocation of STAT and transcriptional activation of immune effector genes.

The potency of the Imd pathway in the anti-P. falciparum response has warranted further molecular dissection in light of recent studies [31, 32, 40]. There are, for example, only a few studies detailing the regulation of Rel2 once it has been translocated to the nucleus. Recent work has demonstrated that the transcription factor Caudal (Cad) is an antagonist of Rel2 (see fig. 1) and also a negative regulator of the Imd pathway's anti-P. falciparum defense in the Anopheles mosquito [41]. Cad was previously identified as a negative regulator of the Imd pathway in adult Drosophila [42].

RNAi-mediated silencing of Cad specifically compromised $P$. falciparum development in the gut tissue, suppressed the midgut microflora and enhanced resistance to systemic bacterial infections, most likely by causing an increased transcriptional abundance of AMPs and other effector genes. Interestingly, $\mathrm{Cad}$ gene silencing resulted 
in increased longevity in the female adult mosquitoes, but the silencing of Cad impaired the mosquito's fecundity and fertility [41], indicating that Cad may display functional diversity in terms of immunity, development and perhaps other processes.

Another study has implicated the transcriptional mediators Kohtalo, Kto and Skuld, Skd as participants in the regulation of the Imd pathway's anti- $P$. falciparum defense in An. gambiae. Depletion of the Kto and Skd genes by RNAi in the mosquito resulted in an increased susceptibility to bacterial and human malaria parasite infection, but not to infection with the rodent malaria parasite P. berghei [43].

\section{The JAK-STAT Pathway}

Little is known about the role of JAK-STAT in insects; however, in Drosophila, this pathway is involved in a variety of developmental processes. It has also been implicated in antibacterial and antiviral defense in Drosophila and the Aedes mosquito [22, 44, 45]. Recent studies in Anopheles have also linked this pathway to anti-Plasmodium defense [46-48]. In Drosophila, this signaling pathway is initiated by the binding of the cytokine ligand Unpaired (UPD) to the transmembrane receptor Domeless (DOME), leading to the phosphorylation of DOME by the JAK tyrosine kinase Hopscotch (HOP; fig. 1). Phosphorylation of DOME recruits a STAT, which is then phosphorylated, dimerized and translocated to the nucleus, where it transcriptionally upregulates immune effector genes. This pathway is tightly regulated by proteins such as the suppressor of cytokine signaling (SOCS) and the protein inhibitor of activated STAT (PIAS). SOCS is transcriptionally activated by this pathway as part of a negative feedback loop that regulates STAT signaling by preventing STAT phosphorylation. PIAS inhibits signaling by binding to STAT proteins and targeting them for degradation $[49,50]$. There are two STAT genes in An. gambiae (STAT1/AgSTAT-B and STAT2/AgSTAT-A) and only one in Drosophila (Stat92E); a one-to-one orthology relationship exists for JAK and DOME in these two species $[24,51]$ (fig. 1).

The JAK-STAT pathway mediates immunity against the malaria parasite through both STAT genes, AgSTAT$A$ and AgSTAT-B. AgSTAT- $A$ is an ancestral gene regulated at the mRNA level by the AgSTAT-B gene. AgSTATA has recently been shown to mediate the transcriptional activation of NO synthase (NOS), which is induced in response to Plasmodium infection and leads to high levels of reactive NO, thereby diminishing parasite development. AgSTAT-A also activates the transcription of SOCS. Silencing of AgSTAT-A increases mature oocyst development in $P$. berghei and P. falciparum-infected mosquitoes [46]. These findings suggest that the JAKSTAT pathway regulates NOS expression and induces immunity to the later oocyst stages of Plasmodium in the An. gambiae midgut. However, Bahia et al. [47] have recently shown that the JAK-STAT pathway controls the early stages of infection with $P$. vivax, another virulent form of human malaria, in the Brazilian malaria vector A. aquasalis.

While the Toll, Imd and JAK-STAT are the best characterized pathways, other pathways have also been shown to play key roles in antiplasmodial immunity such as the insulin/insulin growth factor-1 signaling (IIS) pathway. The activation of the IIS pathway increases susceptibility to $P$. falciparum in An. stephensi and may even alter NF- $\mathrm{BB}$-dependent immunity [52-54].

\section{Anopheles Molecular Immune Responses to Plasmodium Infection}

The past 20 years have witnessed great progress in understanding the mosquito's immune system, and a variety of putative immune genes/effectors have been implicated in the defense against Plasmodium [reviewed in 22, 51]. In particular, ookinete invasion of the midgut epithelium by different Plasmodium species results in the elicitation of both common and diverse molecular responses [7, 34, 55]. These global transcriptomic analyses have identified a plethora of genes that were later shown to represent key players in anti-Plasmodium defense.

One of the first anti-Plasmodium factors studied was the hemocyte-specific thioester complement-like protein TEP1, which binds to and mediates killing of $P$. berghei ookinetes. TEP1 is upregulated $24 \mathrm{~h}$ after ingestion of either P. berghei- or P. falciparum-infected blood and plays a role in the defense against both Plasmodium spp. [31, $34,40,56]$. Two leucine-rich repeat (LRR) proteins, LRIM1 and APL1C, are factors that function with TEP1 to regulate Plasmodium loads in the mosquito. Together, these three factors establish a complement-like pathway that is pivotal for antiplasmodial defense [39]. This defense mechanism is discussed in greater detail later in this review.

In An. gambiae, the superfamily of LRR domain-containing proteins is a gene family that encodes secreted, membrane-bound or cytoplasmic proteins with diverse functions; LRR immune proteins (LRIM) are members within this superfamily and have been shown to be prominent players in the antiplasmodial response $[36,57]$. 
LRIM1 is upregulated in An. gambiae after infection with Plasmodium [34]. Additionally, LRIM1 is a key antagonist of $P$. berghei and causes a substantial majority of the ookinetes to be killed while traversing the midgut, before oocyst formation $[21,36]$. Two other LRIM family members, Anopheles Plasmodium-responsive LRR 1 (APL1) and LRRD7, have also been shown to be involved in the defense of both $P$. falciparum and $P$. berghei development in the mosquito $[21,34,35,58]$.

Interestingly, a recent study has shown that the APL1 locus encodes three genes, APL1A, APL1B and APL1C, which share more than $50 \%$ identity at the amino acid level [35]. This locus lies within a quantitative trait locus that confers vector resistance to $P$. falciparum in wild mosquito populations in Africa [17, 58-60]. Rottschaefer et al. [37] recently examined the molecular genetic variation in the APL1 locus in diverse West African collections of An. gambiae, and they found that the APL1 locus is extremely polymorphic. Within these paralogs, the APL1A gene was thought to be involved in the defense against $P$. falciparum through the Imd pathway. In contrast, the same study indicated that the gene APL1C protects the mosquito against the rodent malaria parasites $P$. berghei and $P$. yoelii only through the Toll signaling pathway [33]. Another study of the APL1 genes showed that they behaved differently than reported by Mitri et al. [33]. While the role for APL1 genes in limiting $P$. falciparum infection was confirmed, a significant role for the APL1A gene in the anti-P. falciparum immune response was not apparent. However, silencing of $A P L 1 B$ and $A P L 1 C$ had a significant impact on P. falciparum infection. As earlier stated, the APL1 gene family has exhibited a complex sequence evolution, including an exceptionally high degree of polymorphism [37]. Therefore, although the latter study confirms a role for APL1 gene family members during $P$. falciparum infection, the differences between the two studies may be explained by different versions of APL1 sequences in the used mosquito strains, or the fact that different $P$. falciparum parasite genotypes resulting in different infection intensities were used in the two studies.

Another class of Plasmodium effectors is the c-type lectins (CTLs). Two members of this family, CTL4 and CTLMA2, are present in the hemolymph of An. gambiae and their transcripts are both upregulated $24 \mathrm{~h}$ after blood feeding on $P$. berghei-infected mice [21]. Interestingly, these two CTLs can protect the rodent Plasmodium ookinetes from destruction [21]. CTL4 is also induced by $P$. falciparum-infected blood with noninvading ookinetes, while CTLGA3 is induced by invading $P$. falciparum ookinetes [34]. CTL4 and CTLMA2 are soluble proteins that are secreted in the hemolymph in the form of a disulfide-linked heterodimeric complex (similar to the LRIM1/ APL1C complex which will be discussed later in this review) and protect the mosquito from infection by G- bacteria [61]. This mode of action may provide a link between their role in antibacterial defense and the melanization of $P$. berghei.

Components of the lipid-transporting system, such as apolipophorin and apolipoprotein $\mathrm{D}$ precursors, also have a significant impact on Plasmodium development $[34,62,63]$. An apolipophorin precursor, RFABG, is induced by $P$. berghei invasion [62], and the transcript level of the apolipoprotein D (APOD) gene is increased upon P. falciparum infection [34]. Apolipophorin-III (ApoLpIII) has recently been identified as a player in midgut antiplasmodial defense. ApoLp-III mRNA is strongly expressed in the Anopheles midgut upon $P$. berghei infection; in addition, silencing of the ApoLp-III gene significantly increases $P$. berghei oocyst levels [63]. Work by Rono et al. [64] demonstrated that lipophorin reduces the parasite-killing efficiency of TEP1; however, the absence of lipophorin increased TEP1's efficiency to bind to Plasmodium ookinetes.

The fibrinogen-related proteins (FREPs) are a pattern recognition receptor (PRR) family that also exhibits antiPlasmodium activity. The FREP gene family is significantly expanded in An. gambiae, with 58 members, as compared to 37 members in the mosquito Aedes aegypti and only 14 in D. melanogaster [65-68]. RNAi-mediated gene-silencing assays have indicated that the FBN8, FBN9 and FBN39 genes are involved in the anti-Plasmodium defense; their involvement is specific, with FBN39 regulating only the mosquito's resistance to the human malaria parasite, and FBN9 and FBN8 being induced in response to both $P$. berghei and $P$. falciparum infection [34, $65,69,70]$.

The G- bacteria-binding proteins represent another PRR family that is important in antimalarial defense. GNBPB3 and GNBPB4 are only upregulated after challenge with $P$. berghei, and GNBPB1 is induced only by $P$. falciparum-infected blood [34, 71]. Also, within the class of PRRs in An. gambiae are the splice variants of the An. gambiae Down syndrome cell adhesion molecule gene (AgDscam), which has been shown to protect mosquitoes against challenge with either $P$. berghei or $P$. falciparum $[72,73]$. The AgDscam gene has been identified as a hypervariable PRR with the potential to generate 31,000 alternative splice forms that are responsible for different pathogen interactions and specificities. Specifically, the Imd and Toll pathways mediate AgDscam-mediated spe- 
cies-specific defenses against Plasmodium and bacteria by regulating the alternative splicing of this gene [73]. The Imd pathway-controlled immune-responsive splicing factors Caper and IRSF1 regulate AgDscam splicing and influence anti-Plasmodium defense specificity. Imd pathway activation was also shown to enhance the association of AgDscam with P. falciparum ookinetes in the mosquito midgut epithelium [73].

\section{Anti-Plasmodium Defense Mechanisms}

\section{The 'Time Bomb' Theory}

Midgut invasion by the Plasmodium ookinete does not leave the mosquito unharmed. According to the 'time bomb' theory, a model of the cellular and molecular response of the An. stephensi midgut epithelium to $P$. berghei ookinete invasion, invading ookinetes inflict irreversible damage on the midgut epithelial cells as the parasite moves in order to reach the basal lamina, where it differentiates into an oocyst $[8,74]$. The invaded cells upregulate NOS expression, have fewer microvilli, undergo DNA fragmentation, and possess abnormally shaped nuclei and a remodeled actin cytoskeleton. In addition to causing cellular damage and eliciting molecular responses, the ookinetes also secrete the Pbs21 surface protein and $\mathrm{PbSub} 2$ protease, which may help facilitate the motility of the ookinete as it glides across the epithelium. The defense response of elevated NOS expression (and consequent NO generation) and the initiation of cell death and protrusion create a ticking time bomb and an altogether hostile environment for the traversing ookinete. Thus, the ookinete must move quickly from the damaged cells in order to continue its development in the mosquito midgut. While the majority of studies have been conducted in An. stephensi, NOS expression is known to be elevated in An. gambiae after P. berghei infection [20,75]. Biochemical studies in An. gambiae reveal nitration in Plasmodium-invaded midgut cells to occur as a two-step process in which the induction of NOS expression is followed by peroxidase activity $[76,77]$. Recent work identified heme peroxidase 2, HPX2, and NADPH oxidase 5, NOX5, as mediators of nitration in the An. gambiae midgut epithelium, and demonstrated that epithelial nitration and TEP1-mediated lysis work sequentially to target Plasmodium ookinetes. The authors propose that nitration of ookinetes in the midgut promotes the subsequent activation of the mosquito complement system [78].
Work conducted by Shiao et al. [79] proposes a woundhealing response mechanism to dead or dying ookinetes that has been argued to be in conflict with the 'time bomb' theory. In this study, the authors claim that while the majority of $P$. berghei ookinetes are killed in the extracellular space in An. gambiae, dead or dying ookinetes are surrounded by a polymerized actin zone formed at the basal layer of adjacent midgut epithelial cells. The formation of this zone is strongly linked to the activation of the melanization response (which is discussed later in this review). Furthermore, the study identified two factors controlling the formation of the actin zone and subsequent activation of melanization: the transmembrane receptor frizzled-2 (Fz2) and the guanosine triphosphate-binding protein cell division cycle 42 (Cdc42). Discussed later in this review, RNAi-mediated silencing of these two factors did not affect ookinete survival. Collectively, these results suggest a separation of parasite killing from subsequent reactions manifested by actin zone formation (in this case, the activation of melanization) [79].

\section{The Mosquito Complement System}

The complement cascade in the Anopheles hemolymph has emerged as a key antiplasmodial defense mechanism. As previously discussed, the mosquito complement C3-like protein TEP1 binds to the surface of midgut-invading ookinetes and marks them for killing [38]. TEP1 circulates in the mosquito hemolymph as a full-length protein and a processed form, TEP $1_{\text {cut }}$. Recent studies have independently revealed that the An. gambiae LRR proteins LRIM1 and APL1C are circulated in the hemolymph as a disulfide-linked heterodimer $[36,39]$. This complex interacts with and stabilizes TEP $1_{\text {cut }}$ and is required for TEP1 accumulation on the ookinete surface. These results reveal that the LRIM1/APL1C/TEP $1_{\text {cut }}$ complex functions as a complement-like system for parasite killing. They also indicate a potential role for the LRIM1/APL1C complex in binding multiple targets, as mammalian multisubunit receptors have similarly been shown to robustly activate the complement pathway. The conformational changes in the LRIM1/APL1C complex can then facilitate the recruitment of additional cascade components such as TEP1-activated proteases. A recent paper has suggested that a cleaved form of TEP1 can act as a convertase for the activation of other TEP1 molecules and that the LRIM1/APL1C complex may regulate the formation of this TEP1 convertase [80]. Future in-depth studies of this complex will provide more detailed insight into complement activation and its role in Plasmodium killing. 


\section{Hemocyte-Mediated Defenses}

Insect blood cells known as hemocytes (which are macrophage-like) play a key role in the mosquito innate immune response against pathogens and exist in the insect's open circulatory system. These cells function in defense against pathogens either directly through phagocytosis or indirectly through secretion of effectors such as AMPs, complement-like proteins and effectors of the melanization response [81]. The hemolymph of the An. gambiae adult female contains three hemocyte sub-types: granulocytes, oenocytoids and prohemocytes. These types can be distinguished from one another by morphological and functional markers. Granulocytes function in phagocytosis, oenocytoids play a role in melanization and the prohemocytes are hypothesized to serve as hematopoietic progenitors [82]. Work by King and Hillyer [83] has identified a novel type of hemocytes, known as periostial hemocytes, which surround the heart in order to phagocytose bacterial and Plasmodium pathogens as they flow in the hemolymph, highlighting the interaction among the mosquito innate immune and circulatory systems.

Transcriptomic profiles of adult female An. gambiae hemocytes following bacteria and Plasmodium infection revealed pathogen-specific signatures of gene regulation and expression. Particularly, 4,047 genes were expressed, with 959 genes being differentially expressed following bacteria or Plasmodium challenge [84]. In addition to varied transcriptomic profiles, the number of circulating hemocytes in adult mosquitoes change in response to infection as well as age and physiological state [85-88]. It has been speculated that such changes may be due to a release of sessile hemocytes (hemocytes attached to tissues) or differentiation of the prohemocytes $[82,86,87$, $89,90]$. A recent study that investigated the in vivo distribution of hemocytes in adult An. gambiae demonstrated that the increase and proliferation of circulating hemocytes following infection is primarily due to mitosis in the circulating hemocytes rather than the differentiation of a progenitor cell type [91].

Interestingly, the differentiation of hemocytes has been implicated in facilitating innate immune memory in $A n$. gambiae. It is much understood that the innate immune system is unable to establish memory in a fashion similar to the adaptive immune system (which is not present in insects). However, memory-like responses, termed immune priming, have been described in insects (as well as other invertebrates) [92-96]. Recent work has demonstrated an immune priming mechanism in mosquitoes in response to Plasmodium in the presence of their midgut microbiota. Particularly, this memory was shown to be primed by the invasion of the mosquito midgut by ookinetes. Ookinete invasion resulted in a long-lasting increase in granulocytes and enhanced immunity to bacteria. This enhanced antibacterial immunity indirectly reduced Plasmodium parasite survival upon reinfection [86].

While the three sub-types of hemocytes are much agreed upon, the number of circulating hemocytes within the adult mosquito is still a source of debate. For example, the authors of the aforementioned study based their conclusions on mosquitoes containing an estimated range of 30,000-50,000 circulating hemocytes [86]. However, other studies have determined that the range of circulating hemocytes in adult mosquitoes and Drosophila flies is between 1,000 and 5,000 [82, 85, 87-89, 91, 97]. Such discrepancies may provide an impetus to further investigate the basic aspects of hemocyte biology in addition to the methods employed to isolate and count hemocytes.

\section{Melanization in the Anti-Plasmodium Defense \\ Response}

Melanization is another innate immune response in the mosquito that the Plasmodium parasite may face. This innate immune mechanism has been genetically mapped to three quantitative trait loci in An. gambiae, collectively called the Plasmodium encapsulation genes: Pen1, Pen 2 and Pen $3[98,99]$. Melanin formation in the mosquito is a result of the proteolytic activation of prophenoloxidase (PPO) to phenoloxidase (PO), induced by a cascade of CLIP serine proteases. PO then oxidizes tyrosine and 3,4-dihydroxy phenylalanine to form reactive quinones that produce melanin. When a pathogen invades the mosquito, the mosquito deposits melanin, which then crosslinks proteins and forms a capsule around the parasite [reviewed in 100]. The melanization process is highly regulated by serine protease inhibitors, or serpins (SRPNs), which block the activation of PO [reviewed in 101].

Genetically selected refractory (R) and susceptible (S) strains of $A n$. gambiae have provided valuable insight into the mosquito's melanization mechanism. The $\mathrm{R}$ strain is highly efficient at blocking Plasmodium development in the midgut via melanization, when compared to the $S$ strain. We will briefly highlight the use of $R$ and $S$ mosquito strains in providing insight about the roles of CLIP serine proteases in melanization.

Silencing of the CLIPA8 gene in $\mathrm{R}$ and in $\mathrm{S}$ mosquitoes in which the anti-Plasmodium gene CTL4 has also been silenced has demonstrated that this CLIP protease is essential for activating the PO cascade and hence necessary for the melanization of $P$. berghei ookinetes [102]. Recent 
work by Yassine et al. [103] has also demonstrated the importance of CLIPA8 in the melanization response against the entomopathogenic fungus Beauveria bassiana in An. gambiae mosquitoes. CLIPA2, A5 and A7 suppress melanization, with CLIPA2 and CLIPA5 acting synergistically to block ookinete invasion. Two CLIPBs, CLIPB14 and CLIPB15, are also involved in the killing of Plasmodium ookinetes and participate in the defense against Gbacteria [104]. CLIPB3, B4, B8 and B17 promote ookinete invasion $[101,102]$, and silencing of the SRPN2 gene increases melanization and reduces the ability of $P$. berghei ookinetes to invade the midgut epithelium [105]. While depletion of the SRPN2 gene was shown to negatively affect the ability of the parasite to invade the midgut epithelium and develop into oocysts, gene silencing of SRPN2 in An. gambiae mosquitoes originally from Cameroon was not found to influence the development of field strains of P. falciparum [106]. The results of this study suggest that some strains of the parasite are efficient at evading the mosquito's innate immune system.

One study has recently demonstrated that CLIPB9 acts as a PPO-activating proteinase that is inhibited by SRPN2. It also showed that CLIPB9 and SRPN2 not only interact to form a regulatory unit of melanization but also affect the life span of adult female mosquitoes [107]. Another SRPN, SRPN6, mediates the defense against malaria parasites and bacteria. In particular, SRPN6 gene expression is induced upon infection with E. coli and both rodent and human malaria parasites, and is specifically expressed in midgut cells invaded by ookinetes and in surrounding hemocytes. Silencing of SRPN6 in An. gambiae has demonstrated that its role in parasite clearance is to inhibit melanization in order to promote parasite lysis [108]. Additionally, silencing of SRPN6 also reduces sporozoite numbers in the salivary glands [109]. A recent study has shown that the LPSinduced TNFa transcription factor-like 3 in An. gambiae is capable of modulating SRPN6 gene expression to influence its anti-Plasmodium response [110].

In addition to the aforementioned melanization effectors, other molecules and anti-Plasmodium factors also modulate the mosquito's melanization response. In $\mathrm{R}$ females, dead Plasmodium ookinetes have been shown to associate with a zone of actin in nearby midgut cells and with melanin deposition on the ookinete surface [79]. As discussed earlier in this review, the genes $F z 2$ and $C d c 42$ are required for these two processes of actin polymerization and melanization [79]. However, RNAi-mediated silencing of these two genes does not affect the killing of Plasmodium ookinetes; rather, these two factors contribute to the mosquito's wound-healing mechanism during
Plasmodium infection. Additionally, gene silencing of CTL4 and CTLMA2 resulted in increased ookinete melanization [21].

TEP1 may also play a role in Plasmodium melanization, since the RNAi-mediated silencing of TEP1 renders $\mathrm{R}$ females unable to melanize $P$. berghei, thereby making them susceptible to infection [38]. However, silencing TEP1 in S mosquitoes increased the number of developing parasites. The results from this work suggest that TEP1-dependent parasite killing is followed by a TEP1independent clearance of dead parasites by lysis and/or melanization [38]. TEP1 has also been implicated in the melanization response to fungal infection in An. gambiae mosquitoes and Sephadex beads $[103,111]$.

Silencing of the complement-like system genes LRIM1 and $A P L 1 C$ also results in a decrease in melanized Plasmodium parasites [36]. Work by Warr et al. [111] has also indicated that the silencing of LRIM1 and TEP1 (as earlier mentioned) compromises the mosquito's ability to melanize Sephadex beads, whereas silencing of CTL4 and CTLMA2 did not affect bead melanization.

Recent work has shown that some strains of P. falciparum are able to evade this complement-like system (i.e. TEP1, LRIM1 and APL1C) in An. gambiae. In particular, this work demonstrated that the silencing of TEP1, LRIM1 and APL1C in An. gambiae prevented the melanization of the Brazilian P. falciparum 7G8 line. However, there was no effect on infection intensity when the African P. falciparum strain NF54 was used, suggesting this line is able to evade this complement-like system. When An. gambiae R mosquitoes were coinfected with 7G8 and another African P. falciparum strain, 3D7, mixed infections comprised of both live and encapsulated parasites were produced in the midgut, suggesting that survival is parasite-specific in nature [112]. Silencing of Rel2 and PGRP-LC led to melanization of Plasmodium in the mosquito midgut, suggesting that the Imd pathway is a negative regulator of the melanization response in the mosquito $[27,30,113]$.

\section{Mosquito-Bacteria Interactions}

\section{The Mosquito Midgut Microbiota}

The presence of bacteria in the midgut (the midgut microbiota) stimulates a basal innate immune activity consisting of the induction of AMPs and other immunespecific genes that act against Plasmodium and prime the mosquito for infection [114]. In this study, mosquitoes possessing their midgut microbiota were also shown to have upregulated key antibacterial and anti-Plasmodium 
factors, whereas mosquitoes treated with antibiotics that eliminate the majority of the midgut microbiota did not show this upregulation profile and were more susceptible to Plasmodium infection. Cofeeding mosquitoes with bacteria and $P$. falciparum gametocytes also resulted in the elicitation of an immune response and resistance to infection. Work by Meister et al. [113] has suggested that the PRR molecule known as long peptidoglycan recognition protein C, PGRP-LC, which activates the Imd pathway in the mosquito in response to bacteria, modulates Plasmodium infection by controlling the microbial flora in the mosquito midgut. Numerous surveys of mosquito midgut microbiota in laboratory and wild mosquitoes have been performed, and common bacterial genera (Asaia, Enterobacter, Pseudomonas, Pantoea and others) have been identified, with some of these bacteria being closely associated with Anopheles mosquitoes. For example, the acetic acid bacteria Asaia has emerged as an important symbiont of Anopheles [115]. However, it is not clear if Asaia can directly reduce Plasmodium infection.

Several studies have shown that the mosquito midgut microbiota negatively affect the ability of Plasmodium parasites to develop to the oocyst stage in the mosquito gut tissue [1, 116-119]. A number of bacterial species have also been shown to produce potential antimalarial compounds [120], but the effects on mosquito-stage Plasmodium development have not yet been examined. Bacteria may play an indirect role in parasite interference through the induction of an anti-Plasmodium immune response in the midgut, as discussed earlier.

Recent work by Kumar et al. [121] has revealed a peroxidase/dual oxidase system that forms a dityrosine network in the midgut and decreases the permeability of the midgut to immune activators, protecting the microbiota and also providing a safe environment for Plasmodium to develop in the midgut. Dual oxidase, Duox, is a transmembrane protein that produces the hydrogen peroxide substrate for peroxidase. RNAi-mediated silencing of the heme peroxidase-immunomodulatory peroxidase, IMPer, gene has been shown to result in decreased bacterial load in the midgut and induced the upregulation of key antibacterial effectors such as cecropin and PGRPLB. IMPer gene silencing also reduced $P$. berghei ookinete and oocyst development via the induction of NOS in antibiotic-treated (also called aseptic) An. gambiae female mosquitoes; in addition, through the induction of NOS, RNAi-mediated silencing of the IMPer gene also reduced the development of P. falciparum in An. stephensi and An. gambiae females possessing their microbiota as well in females with decreased microbiota load via treatment with antibiotics. RNAi-mediated silencing of the Duox gene also reduced $P$. falciparum in An. gambiae via NOS induction. Hence, this complex when intact appears to block midgut immune responses to bacteria and Plasmodium, allowing proliferation and development.

Although the absolute mechanism by which bacteria inhibit Plasmodium is as yet unclear, their potential usefulness as a biologically based control strategy is apparent. A recent study has demonstrated engineered mosquito midgut microbiota potential as a control strategy. In this study, the investigators developed a strategy to engineer symbiotic bacteria to deliver antimalarial effector molecules to the midgut lumen, thereby rendering the mosquitoes resistant to Plasmodium infection [122].

\section{Antibacterial Effectors}

As earlier noted, the mosquito employs antibacterial effectors to battle the malaria parasite. We have discussed some of these effectors earlier in this review with regards to their antiplasmodial roles. In this section, we will briefly highlight some of these effectors' roles in the antibacterial response.

The complement-like protein TEP1 is involved in the bacterial phagocytosis response and has been shown to bind to both G- and G+ bacteria [123]. The mosquito PRR $A g$ Dscam is a determinant of resistance and bacterial phagocytosis and also modulates the mosquito's response to Plasmodium infection $[72,73]$. FREP FBN9 interacts with G- and G+ bacteria and appears to form dimers in order to specifically bind to bacterial surfaces with different affinities [65]. This FREP may use a multimerization mechanism similar to that of LRIM1/APL1C (earlier discussed in detail) and may dimerize with other FREPs, thereby providing diverse PAMP interaction specificities, as a means of increasing the mosquito's PRR repertoire. Whether FBN9 also forms dimers when binding to human and rodent malaria parasites remains unknown; however, direct interaction is thought to occur, as implied by FBN9's interaction with bacteria [65]. The PRR GNBPB4 is known to interact with a wide range of pathogens. Particularly, GNBPB4 has been shown to directly interact with $E$. coli and colocalize with $P$. berghei ookinetes [124].

Two other immune-responsive factors involved in the mosquito's antibacterial and antimalarial responses are the Rel2-S and Rel2-F isoforms of the Rel2 gene. These isoforms not only modulate the defense against $\mathrm{G}-$ and $\mathrm{G}+$ bacteria but also regulate several of the AMPs and antiparasitic genes, as mentioned earlier in this review. The immunoglobulin superfamily members known as the in- 
fection responsive with immunoglobulin domain (IRID) genes are factors that also participate in the mosquito's antibacterial and antiplasmodial responses, with the IRID6 gene functioning to limit $P$. falciparum as well as bacterial infection [125]. Lysozymes, another class of antimicrobial immune effectors, are also important in the antiplasmodial defense. Lysozyme c-1 (LYSC-1) has recently been shown to act as a protective agonist of the development of $P$. berghei and P. falciparum oocysts. This antimicrobial effector binds directly to Plasmodium oocysts following midgut invasion in An. gambiae [126, 127]. In addition, silencing of the LYSC-1 gene in $A n$. gambiae as well as in the Asian malaria vector An. dirus significantly reduced $P$. berghei infection $[127,128]$.

\section{Concluding Remarks}

The Anopheles mosquito makes use of many weapons to battle Plasmodium. The molecular and cellular events involved in the infection of a mosquito with different
Plasmodium spp. may be quite similar yet also divergent, indicating the great complexity and intricacy of parasite-mosquito interactions. Given the increasing prevalence and spread of malaria, especially in Africa, there is an impetus for further dissection of the innate immune system of Anopheles, with an emphasis on how it modulates and regulates Plasmodium infection. The insight and knowledge gained from such studies can provide the necessary tools for creating antimalarial strategies based on amplifying the mosquito's anti-Plasmodium defenses.

\section{Acknowledgments}

We would like to thank members of the Dimopoulos laboratory. We also gratefully acknowledge support from the National Institutes of Health/National Institute of Allergy and Infectious Disease, a UNCF-Merck Science Initiative Graduate Research Fellowship to A.M.C., and the Bloomberg Family Foundation. We also thank Dr. Deborah McClellan for editorial assistance.

\section{References}

1 Cirimotich CM, Clayton AM, Dimopoulos G: Low- and high-tech approaches to control plasmodium parasite transmission by anopheles mosquitoes. J Trop Med 2011;2011: 891342.

2 Baton LA, Ranford-Cartwright LC: How do malaria ookinetes cross the mosquito midgut wall? Trends Parasitol 2005;21:22-28.

3 Whitten MM, Shiao SH, Levashina EA: Mosquito midguts and malaria: cell biology, compartmentalization and immunology. Parasite Immunol 2006;28:121-130.

4 Sinden RE, Billingsley PF: Plasmodium invasion of mosquito cells: hawk or dove? Trends Parasitol 2001;17:209-212.

5 Baton LA, Ranford-Cartwright LC: Plasmodium falciparum ookinete invasion of the midgut epithelium of Anopheles stephensi is consistent with the time bomb model. Parasitology 2004;129:663-676.

6 Maier WA, Becker-Feldman H, Seitz HM: Pathology of malaria-infected mosquitoes. Parasitol Today 1987;3:216-218.

7 Gupta L, Kumar S, Han YS, Pimenta PF, Barillas-Mury C: Midgut epithelial responses of different mosquito-plasmodium combinations: the actin cone zipper repair mechanism in Aedes aegypti. Proc Natl Acad Sci USA 2005;102:4010-4015.

-8 Han YS, Thompson J, Kafatos FC, BarillasMury C: Molecular interactions between Anopheles stephensi midgut cells and Plasmodium berghei: the time bomb theory of ooki- nete invasion of mosquitoes. EMBO J 2000; 16 Collins FH, Sakai RK, Vernick KD, Paskewitz 19:6030-6040.

-9 Torii M, Nakamura K, Sieber KP, Miller LH, Aikawa M: Penetration of the mosquito ( $A e$ des aegypti) midgut wall by the ookinetes of Plasmodium gallinaceum. J Protozool 1992; 39:449-454.

10 Vernick KD, Fujioka H, Seeley DC, Tandler B, Aikawa M, Miller LH: Plasmodium gallinaceum: a refractory mechanism of ookinete killing in the mosquito, Anopheles gambiae. Exp Parasitol 1995;80:583-595.

11 Vlachou D, Zimmermann T, Cantera R, Janse CJ, Waters AP, Kafatos FC: Real-time, in vivo analysis of malaria ookinete locomotion and mosquito midgut invasion. Cell Microbiol 2004;6:671-685.

12 Zieler H, Dvorak JA: Invasion in vitro of mosquito midgut cells by the malaria parasite proceeds by a conserved mechanism and results in death of the invaded midgut cells. Proc Natl Acad Sci USA 2000;97:1151611521.

13 Levashina EA: Immune responses in Anopheles gambiae. Insect Biochem Mol Biol 2004; 34:673-678.

14 Vlachou D, Schlegelmilch T, Runn E, Mendes A, Kafatos FC: The developmental migration of plasmodium in mosquitoes. Curr Opin Genet Dev 2006;16:384-391.

15 Sinden RE: Molecular interactions between Plasmodium and its insect vectors. Cell Microbiol 2002;4:713-724.
S, Seeley DC, Miller LH, Collins WE, Campbell CC, Gwadz RW: Genetic selection of a plasmodium-refractory strain of the malaria vector anopheles gambiae. Science 1986;234: 607-610.

17 Niare O, Markianos K, Volz J, Oduol F, Toure A, Bagayoko M, Sangare D, Traore SF, Wang R, Blass C, Dolo G, Bouare M, Kafatos FC, Kruglyak L, Toure YT, Vernick KD: Genetic loci affecting resistance to human malaria parasites in a West African mosquito vector population. Science 2002;298:213-216.

18 Hillyer JF, Barreau C, Vernick KD: Efficiency of salivary gland invasion by malaria sporozoites is controlled by rapid sporozoite destruction in the mosquito haemocoel. International Journal for Parasitology 2007;37: 673-681.

19 Alavi Y, Arai M, Mendoza J, Tufet-Bayona M, Sinha R, Fowler K, Billker O, Franke-Fayard B, Janse CJ, Waters A, Sinden RE: The dynamics of interactions between Plasmodium and the mosquito: a study of the infectivity of Plasmodium berghei and Plasmodium gallinaceum, and their transmission by Anopheles stephensi, Anopheles gambiae and Aedes aegypti. Int J Parasitol 2003;33:933-943.

20 Dimopoulos G, Seeley D, Wolf A, Kafatos FC: Malaria infection of the mosquito Anopheles gambiae activates immune-responsive genes during critical transition stages of the parasite life cycle. EMBO J 1998;17:6115-6123. 
-21 Osta MA, Christophides GK, Kafatos FC: Effects of mosquito genes on plasmodium development. Science 2004;303:2030-2032.

-22 Cirimotich CM, Dong Y, Garver LS, Sim S, Dimopoulos G: Mosquito immune defenses against plasmodium infection. Dev Comp Immunol 2010;34:387-395.

23 Michel K, Kafatos FC: Mosquito immunity against plasmodium. Insect Biochem $\mathrm{Mol}$ Biol 2005;35:677-689.

24 Meister S, Koutsos AC, Christophides GK: The plasmodium parasite - a 'new' challenge for insect innate immunity. Int J Parasitol 2004;34:1473-1482.

25 Aggarwal K, Silverman N: Positive and negative regulation of the Drosophila immune response. BMB Rep 2008;41:267-277.

26 Ramirez JL, Dimopoulos G: The toll immune signaling pathway control conserved anti-dengue defenses across diverse Ae. aegypti strains and against multiple dengue virus serotypes. Dev Comp Immunol 2010;34:625-629.

-27 Meister S, Kanzok SM, Zheng XL, Luna C, Li TR, Hoa NT, Clayton JR, White KP, Kafatos FC, Christophides GK, Zheng L: Immune signaling pathways regulating bacterial and malaria parasite infection of the mosquito anopheles gambiae. Proc Natl Acad Sci USA 2005;102:11420-11425.

28 Vizioli J, Bulet P, Hoffmann JA, Kafatos FC, Muller HM, Dimopoulos G: Gambicin: a novel immune responsive antimicrobial peptide from the malaria vector anopheles gambiae. Proc Natl Acad Sci USA 2001;98:1263012635.

-29 Luna C, Hoa NT, Lin H, Zhang L, Nguyen HL, Kanzok SM, Zheng L: Expression of immune responsive genes in cell lines from two different Anopheline species. Insect Mol Biol 2006; 15:721-729.

- 30 Frolet C, Thoma M, Blandin S, Hoffmann JA, Levashina EA: Boosting NF- $\kappa B$-dependent basal immunity of Anopheles gambiae aborts development of plasmodium berghei. Immunity 2006;25:677-685.

- 31 Garver LS, Dong Y, Dimopoulos G: Caspar controls resistance to Plasmodium falciparum in diverse Anopheline species. PLoS Pathog 2009;5:e1000335.

- 32 Garver LS, Bahia AC, Das S, Souza-Neto JA, Shiao J, Dong Y, Dimopoulos G: Anopheles Imd pathway factors and effectors in infection intensity-dependent anti-Plasmodium action. PLoS Pathog 2012;8:e1002737.

-33 Mitri C, Jacques JC, Thiery I, Riehle MM, Xu J, Bischoff E, Morlais I, Nsango SE, Vernick $\mathrm{KD}$, Bourgouin C: Fine pathogen discrimination within the apll gene family protects Anopheles gambiae against human and rodent malaria species. PLoS Pathog 2009;5:e1000576.

- 34 Dong Y, Aguilar R, Xi Z, Warr E, Mongin E, Dimopoulos G: Anopheles gambiae immune responses to human and rodent plasmodium parasite species. PLoS Pathog 2006;2:e52.

- 35 Riehle MM, Xu J, Lazzaro BP, Rottschaefer SM, Coulibaly B, Sacko M, Niare O, Morlais I, Traore SF, Vernick KD: Anopheles gambiae
APL1 is a family of variable LRR proteins required for Rel1-mediated protection from the malaria parasite, Plasmodium berghei. PLoS One 2008;3:e3672.

36 Povelones M, Waterhouse RM, Kafatos FC, Christophides GK: Leucine-rich repeat protein complex activates mosquito complement in defense against plasmodium parasites. Science 2009;324:258-261.

37 Rottschaefer SM, Riehle MM, Coulibaly B, Sacko M, Niare O, Morlais I, Traore SF, Vernick KD, Lazzaro BP: Exceptional diversity, maintenance of polymorphism, and recent directional selection on the apl1 malaria resistance genes of anopheles gambiae. PLoS Biol 2011;9:e1000600.

38 Blandin S, Shiao SH, Moita LF, Janse CJ, Waters AP, Kafatos FC, Levashina EA: Complement-like protein TEP1 is a determinant of vectorial capacity in the malaria vector Anopheles gambiae. Cell 2004;116:661-670.

- 39 Fraiture M, Baxter RH, Steinert S, Chelliah Y, Frolet C, Quispe-Tintaya W, Hoffmann JA, Blandin SA, Levashina EA: Two mosquito LRR proteins function as complement control factors in the TEP1-mediated killing of plasmodium. Cell Host Microbe 2009;5:273-284.

40 Dong Y, Das S, Cirimotich C, Souza-Neto JA, McLean KJ, Dimopoulos G: Engineered anopheles immunity to plasmodium infection. PLoS Pathog 2011;7:e1002458.

41 Clayton AM, Cirimotich CM, Dong Y, Dimopoulos G: Caudal is a negative regulator of the Anopheles IMD pathway that controls resistance to Plasmodium falciparum infection. Dev Comp Immunol 2013;39:323-332.

42 Ryu J-H, Kim S-H, Lee H-Y, Bai JY, Nam Y-D, Bae J-W, Lee DG, Shin SC, Ha E-M, Lee W-J: Innate immune homeostasis by the homeobox gene Caudal and commensal-gut mutualism in Drosophila. Science 2008;319: 777-782.

43 Chen Y, Dong Y, Sandiford S, Dimopoulos G: Transcriptional mediators Kto and Skd are involved in the regulation of the IMD pathway and anti-Plasmodium defense in Anopheles gambiae. PLoS One 2012;7:e45580.

-44 Dostert C, Jouanguy E, Irving P, Troxler L, Galiana-Arnoux D, Hetru C, Hoffmann JA, Imler JL: The Jak-STAT signaling pathway is required but not sufficient for the antiviral response of drosophila. Nat Immunol 2005;6:946-953.

45 Souza-Neto JA, Sim S, Dimopoulos G: An evolutionary conserved function of the JakSTAT pathway in anti-dengue defense. Proc Natl Acad Sci USA 2009;106:17841-17846.

46 Gupta L, Molina-Cruz A, Kumar S, Rodrigues J, Dixit R, Zamora RE, Barillas-Mury C: The STAT pathway mediates late-phase immunity against Plasmodium in the mosquito Anopheles gambiae. Cell Host Microbe 2009;5:498507.

47 Bahia AC, Kubota MS, Tempone AJ, Araujo HR, Guedes BA, Orfano AS, Tadei WP, RiosVelasquez CM, Han YS, Secundino NF, Barillas-Mury C, Pimenta PF, Traub-Cseko YM: The JAK-STAT pathway controls Plasmodium vivax load in early stages of Anopheles aquasalis infection. PLoS Negl Trop Dis 2011;5:e1317.

-48 Barillas-Mury C, Han Y-S, Seeley D, Kafatos FC: Anopheles gambiae Ag-STAT, a new insect member of the STAT family, is activated in response to bacterial infection. EMBO J 1999;18:959-967.

49 Agaisse H, Perrimon N: The roles of JAK/ STAT signaling in Drosophila immune responses. Immunol Rev 2004;198:72-82.

50 Wormald S, Hilton DJ: Inhibitors of cytokine signal transduction. J Biol Chem 2004;279: 821-824.

51 Christophides GK, Vlachou D, Kafatos FC: Comparative and functional genomics of the innate immune system in the malaria vector Anopheles gambiae. Immunol Rev 2004;198: 127-148.

52 Luckhart S, Riehle MA: The insulin signaling cascade from nematodes to mammals: insights into innate immunity of Anopheles mosquitoes to malaria parasite infection. Dev Comp Immunol 2007;31:647-656.

- 53 Pakpour N, Corby-Harris V, Green GP Smithers HM, Cheung KW, Riehle MA, Luckhart S: Ingested human insulin inhibits the mosquito NF- $\mathrm{kB}$-dependent immune response to Plasmodium falciparum. Infect Immun 2012;80:2141-2149.

54 Drexler A, Nuss A, Hauck E, Glennon E, Cheung K, Brown M, Luckhart S: Human IGF1 extends lifespan and enhances resistance to Plasmodium falciparum infection in the malaria vector anopheles stephensi. J Exp Biol 2013;216:208-217.

-55 Tahar R, Boudin C, Thiery I, Bourgouin C: Immune response of Anopheles gambiae to the early sporogonic stages of the human malaria parasite Plasmodium falciparum. EMBO J 2002;21:6673-6680.

56 Blandin SA, Marois E, Levashina EA: Antimalarial responses in Anopheles gambiae: from a complement-like protein to a complement-like pathway. Cell Host Microbe 2008;3:364-374.

57 Waterhouse RM, Povelones M, Christophides GK: Sequence-structure-function relations of the mosquito leucine-rich repeat immune proteins. BMC Genomics 2010;11:531.

58 Riehle MM, Markianos K, Niare O, Xu J, Li J, Toure AM, Podiougou B, Oduol F, Diawara S, Diallo M, Coulibaly B, Ouatara A, Kruglyak L, Traore SF, Vernick KD: Natural malaria infection in Anopheles gambiae is regulated by a single genomic control region. Science 2006; 312:577-579.

59 Menge DM, Zhong D, Guda T, Gouagna L, Githure J, Beier J, Yan G: Quantitative trait loci controlling refractoriness to Plasmodium falciparum in natural Anopheles gambiae mosquitoes from a malaria-endemic region in western Kenya. Genetics 2006;173:235-241.

60 Riehle MM, Markianos K, Lambrechts L, Xia A, Sharakhov I, Koella JC, Vernick KD: A major genetic locus controlling natural Plasmodium falciparum infection is shared by East and West African Anopheles gambiae. Malar J 2007;6:87.
Anopheles Innate Immune System in the Defense against Malaria Infection 
61 Schnitger AK, Yassine H, Kafatos FC, Osta MA: Two C-type lectins cooperate to defend Anopheles gambiae against Gram-negative bacteria. J Biol Chem 2009;284:17616-17624.

-62 Vlachou D, Schlegelmilch T, Christophides GK, Kafatos FC: Functional genomic analysis of midgut epithelial responses in Anopheles during Plasmodium invasion. Curr Biol 2005; 15:1185-1195.

-63 Gupta L, Noh JY, Jo YH, Oh SH, Kumar S, Noh MY, Lee YS, Cha SJ, Seo SJ, Kim I, Han YS, Barillas-Mury C: Apolipophorin-III mediates antiplasmodial epithelial responses in Anopheles gambiae (G3) mosquitoes. PLoS One 2010;5:e15410.

-64 Rono MK, Whitten MM, Oulad-Abdelghani M, Levashina EA, Marois E: The major yolk protein vitellogenin interferes with the antiplasmodium response in the malaria mosquito Anophelesgambiae. PLoS Biol2010;8:e1000434.

-65 Dong Y, Dimopoulos G: Anopheles fibrinogenrelated proteins provide expanded pattern recognition capacity against bacteria and malaria parasites. J Biol Chem 2009;284:9835-9844.

66 Wang X, Zhao Q, Christensen BM: Identification and characterization of the fibrinogenlike domain of fibrinogen-related proteins in the mosquito, Anopheles gambiae, and the fruitfly, Drosophila melanogaster, genomes. BMC Genomics 2005;6:114.

67 Middha S, Wang X: Evolution and potential function of fibrinogen-like domains across twelve Drosophila species. BMC Genomics 2008;9:260.

68 Waterhouse RM, Kriventseva EV, Meister S, Xi Z, Alvarez KS, Bartholomay LC, BarillasMury C, Bian G, Blandin S, Christensen BM, Dong Y, Jiang H, Kanost MR, Koutsos AC, Levashina EA, Li J, Ligoxygakis P, Maccallum RM, Mayhew GF, Mendes A, Michel K, Osta MA, Paskewitz S, Shin SW, Vlachou D, Wang L, Wei W, Zheng L, Zou Z, Severson DW, Raikhel AS, Kafatos FC, Dimopoulos G, Zdobnov EM, Christophides GK: Evolutionary dynamics of immune-related genes and pathways in disease-vector mosquitoes. Science 2007;316:1738-1743.

-69 Dimopoulos G, Casavant TL, Chang S, Scheetz T, Roberts C, Donohue M, Schultz J, Benes V, Bork P, Ansorge W, Soares MB, Kafatos FC: Anopheles gambiae pilot gene discovery project: identification of mosquito innate immunity genes from expressed sequence tags generated from immune-competent cell lines. Proc Natl Acad Sci USA 2000;97:66196624.

-70 Dimopoulos G, Christophides GK, Meister S, Schultz J, White KP, Barillas-Mury C, Kafatos FC: Genome expression analysis of Anopheles gambiae: responses to injury, bacterial challenge, and malaria infection. Proc Natl Acad Sci USA 2002;99:8814-8819.

-71 Dimopoulos G, Richman A, Muller HM, Kafatos FC: Molecular immune responses of the mosquito Anopheles gambiae to bacteria and malaria parasites. Proc Natl Acad Sci USA 1997;94:11508-11513.
72 Dong Y, Taylor HE, Dimopoulos G: AgDscam, a hypervariable immunoglobulin domaincontaining receptor of the Anopheles gambiae innate immune system. PLoS Biol 2006;4:e229.

73 Dong Y, Cirimotich CM, Pike A, Chandra R, Dimopoulos G: Anopheles NF-kB-regulated splicing factors direct pathogen-specific repertoires of the hypervariable pattern recognition receptor AgDscam. Cell Host Microbe 2012;12:521-530.

74 Han YS, Barillas-Mury C: Implications of time bomb model of ookinete invasion of midgut cells. Insect Biochem Mol Biol 2002; 32:1311-1316.

75 Luckhart S, Vodovotz Y, Cui L, Rosenberg R: The mosquito Anopheles stephensi limits malaria parasite development with inducible synthesis of nitric oxide. Proc Natl Acad Sci USA 1998;95:5700-5705.

76 Kumar S, Gupta L, Han YS, Barillas-Mury C: Inducible peroxidases mediate nitration of anopheles midgut cells undergoing apoptosis in response to plasmodium invasion. J Biol Chem 2004;279:53475-53482.

77 Kumar S, Barillas-Mury C: Ookinete-induced midgut peroxidases detonate the time bomb in anopheline mosquitoes. Insect Biochem Mol Biol 2005;35:721-727.

78 Oliveira Gde A, Lieberman J, Barillas-Mury C: Epithelial nitration by a peroxidase/NOX5 system mediates mosquito antiplasmodial immunity. Science 2012;335:856-859.

79 Shiao SH, Whitten MM, Zachary D, Hoffmann JA, Levashina EA: Fz2 and Cdc42 mediate melanization and actin polymerization but are dispensable for plasmodium killing in the mosquito midgut. PLoS Pathog 2006; 2:e133.

80 Baxter RH, Steinert S, Chelliah Y, Volohonsky G, Levashina EA, Deisenhofer J: A heterodimeric complex of the LRR proteins LRIM1 and APL1C regulates complementlike immunity in Anopheles gambiae. Proc Natl Acad Sci USA 2010;107:16817-16822.

81 Lavine MD, Strand MR: Insect hemocytes and their role in immunity. Insect Biochem Mol Biol 2002;32:1295-1309.

82 Castillo J, Robertson A, Strand M: Characterization of hemocytes from the mosquitoes Anopheles gambiae and Aedes aegypti. Insect Biochem Mol Biol 2006;36:891-903.

83 King JG, Hillyer JF: Infection-induced interaction between the mosquito circulatory and immune systems. PLoS Pathog 2012;8:e1003058.

84 Baton LA, Robertson A, Warr E, Strand MR, Dimopoulos G: Genome-wide transcriptomic profiling of anopheles gambiae hemocytes reveals pathogen-specific signatures upon bacterial challenge and Plasmodium berghei infection. BMC Genomics 2009;10:257.

85 Coggins S, Estevez-Lao T, Hillyer J: Increased survivorship following bacterial infection by the mosquito Aedes aegypti as compared to Anopheles gambiae correlates with increased transcriptional induction of antimicrobial peptides. Dev Comp Immunol 2012;37:390401.
86 Rodrigues J, Brayner FA, Alves LC, Dixit R, Barillas-Mury C: Hemocyte differentiation mediates innate immune memory in Anopheles gambiae mosquitoes. Science 2010;329: 1353-1355.

87 Castillo J, Brown M, Strand M: Blood feeding and insulin-like peptide 3 stimulate proliferation of hemocytes in the mosquito Aedes aegypti. PLoS Pathog 2011;7:e1002274.

88 Hillyer J, Schmidt S, Fuchs J, Boyle J, Christensen B: Age-associated mortality in immune challenged mosquitoes (Aedes aegypti) correlates with a decrease in haemocyte numbers. Cell Microbiol 2005;7:39-51.

89 Markus R, Laurinyecz B, Kurucz E, Honti V, Bajusz I, Sipos B, Somogyi K, Kronhamn J, Hultmark D, Ando I: Sessile hemocytes as a hematopoietic compartment in Drosophila melanogaster. Proc Natl Acad Sci USA 2009; 106:4805-4809.

90 Ratcliffe N, Rowley A: A comparative synopsis of the structure and function of the blood cells of insects and other invertebrates. Dev Comp Immunol 1979;3:189-221.

91 King J, Hillyer J: Spatial and temporal in vivo analysis of circulating and sessile immune cells in mosquitoes: hemocyte mitosis following infection. BMC Biology 2013;11:55.

92 Roth O, Sadd BM, Schmid-Hempel P, Kurtz $\mathrm{J}$ : Strain-specific priming of resistance in the red flour beetle, Tribolium castaneum. Proc Biol Sci 2009;276:145-151.

93 Pham LN, Dionne MS, Shirasu-Hiza M, Schneider DS: A specific primed immune response in Drosophila is dependent on phagocytes. PLoS Pathog 2007;3:e26.

$\$ 94$ Sadd BM, Schmid-Hempel P: Insect immunity shows specificity in protection upon secondary pathogen exposure. Curr Biol 2006;16:1206-1210.

95 Schmid-Hempel P: Evolutionary ecology of insect immune defenses. Annu Rev Entomol 2005;50:529-551.

$\$ 96$ Moret Y, Siva-Jothy MT: Adaptive innate immunity? Responsive-mode prophylaxis in the mealworm beetle, Tenebrio molitor. Proc Biol Sci 2003;270:2475-2480.

97 Lanot R, Zachary D, Holder F, Meister M: Postembryonic hematopoiesis in Drosophila. Dev Biol 2001;230:243-257.

98 Zheng L, Wang S, Romans P, Zhao H, Luna C, Benedict MQ: Quantitative trait loci in Anopheles gambiae controlling the encapsulation response against Plasmodium cynomolgi Ceylon. BMC Genet 2003;4:16.

99 Zheng L, Cornel AJ, Wang R, Erfle H, Voss $\mathrm{H}$, Ansorge W, Kafatos FC, Collins FH: Quantitative trait loci for refractoriness of Anopheles gambiae to Plasmodium cynomolgi B. Science 1997;276:425-428.

100 Nappi AJ, Christensen BM: Melanogenesis and associated cytotoxic reactions: applications to insect innate immunity. Insect Biochem Mol Biol 2005;35:443-459.

101 Barillas-Mury C: Clip proteases and plasmodium melanization in Anopheles gambiae. Trends Parasitol 2007;23:297-299. 
102 Volz J, Muller HM, Zdanowicz A, Kafatos FC, Osta MA: A genetic module regulates the melanization response of anopheles to plasmodium. Cell Microbiol 2006;8:13921405.

103 Yassine H, Kamareddine L, Osta MA: The mosquito melanization response is implicated in defense against the entomopathogenic fungus Beauveria bassiana. PLoS Pathog 2012;8:e1003029.

104 Volz J, Osta MA, Kafatos FC, Muller HM: The roles of two clip domain serine proteases in innate immune responses of the malaria vector Anopheles gambiae. J Biol Chem 2005;280:40161-40168.

105 Michel K, Budd A, Pinto S, Gibson TJ, Kafatos FC: Anopheles gambiae SRPN2 facilitates midgut invasion by the malaria parasite Plasmodium berghei. EMBO Rep 2005;6: 891-897.

106 Michel K, Suwanchaichinda C, Morlais I, Lambrechts L, Cohuet A, Awono-Ambene PH, Simard F, Fontenille D, Kanost MR, Kafatos FC: Increased melanizing activity in Anopheles gambiae does not affect development of Plasmodium falciparum. Proc Natl Acad Sci USA 2006;103:16858-16863.

107 An C, Budd A, Kanost MR, Michel K: Characterization of a regulatory unit that controls melanization and affects longevity of mosquitoes. Cell Mol Life Sci 2011;68:19291939.

-108 Abraham EG, Pinto SB, Ghosh A, Vanlandingham DL, Budd A, Higgs S, Kafatos FC, Jacobs-Lorena M, Michel K: An immuneresponsive serpin, SRPN6, mediates mosquito defense against malaria parasites. Proc Natl Acad Sci USA 2005;102:16327-16332.

-109 Pinto SB, Kafatos FC, Michel K: The parasite invasion marker SRPN6 reduces sporozoite numbers in salivary glands of Anopheles gambiae. Cell Microbiol 2008;10:891-898.

-110 Smith RC, Eappen AG, Radtke AJ, JacobsLorena M: Regulation of anti-plasmodium immunity by a LITAF-like transcription factor in the malaria vector Anopheles gambiae. PLoS Pathog 2012;8:e1002965.
111 Warr E, Lambrechts L, Koella JC, Bourgouin C, Dimopoulos G: Anopheles gambiae immune responses to sephadex beads: involvement of anti-plasmodium factors in regulating melanization. Insect Biochem Mol Biol 2006;36:769-778.

112 Molina-Cruz A, DeJong RJ, Ortega C, Haile A, Abban E, Rodrigues J, Jaramillo-Gutierrez G, Barillas-Mury C: Some strains of Plasmodium falciparum, a human malaria parasite, evade the complement-like system of Anopheles gambiae mosquitoes. Proc Natl Acad Sci USA 2012;109:E1957-E1962.

113 Meister S, Agianian B, Turlure F, Relogio A, Morlais I, Kafatos FC, Christophides GK: Anopheles gambiae PGRPLC-mediated defense against bacteria modulates infections with malaria parasites. PLoS Pathog 2009; 5:e1000542.

114 Dong Y, Manfredini F, Dimopoulos G: Implication of the mosquito midgut microbiota in the defense against malaria parasites. PLoS Pathog 2009;5:e1000423.

115 Favia G, Ricci I, Damiani C, Raddadi N, Crotti E, Marzorati M, Rizzi A, Urso R, Brusetti L, Borin S, Mora D, Scuppa P, Pasqualini L, Clementi E, Genchi M, Corona S, Negri I, Grandi G, Alma A, Kramer L, Esposito F, Bandi C, Sacchi L, Daffonchio D: Bacteria of the genus Asaia stably associate with Anopheles stephensi, an Asian malarial mosquito vector. Proc Natl Acad Sci USA 2007; 104:9047-9051.

116 Cirimotich CM, Dong Y, Clayton AM, Sandiford SL, Souza-Neto JA, Mulenga M, Dimopoulos G: Natural microbe-mediated refractoriness to plasmodium infection in Anopheles gambiae. Science 2011;332:855858.

-117 Cirimotich CM, Ramirez JL, Dimopoulos G: Native microbiota shape insect vector competence for human pathogens. Cell Host Microbe 2011;10:307-310.

118 Pumpuni CB, Beier MS, Nataro JP, Guers LD, Davis JR: Plasmodium falciparum: inhibition of sporogonic development in Anopheles stephensi by Gram-negative bacteria. Exp Parasitol 1993;77:195-199.

119 Gonzalez-Ceron L, Santillan F, Rodriguez MH, Mendez D, Hernandez-Avila JE: Bacteria in midguts of field-collected Anopheles albimanus block Plasmodium vivax sporogonic development. J Med Entomol 2003;40: 371-374.
120 Azambuja P, Garcia ES, Ratcliffe NA: Gut microbiota and parasite transmission by insect vectors. Trends Parasitol 2005;21:568572.

121 Kumar S, Molina-Cruz A, Gupta L, Rodrigues J, Barillas-Mury C: A peroxidase/ dual oxidase system modulates midgut epithelial immunity in Anopheles gambiae. Science 2010;327:1644-1648.

122 Wang S, Ghosh AK, Bongio N, Stebbings KA, Lampe DJ, Jacobs-Lorena M: Fighting malaria with engineered symbiotic bacteria from vector mosquitoes. Proc Natl Acad Sci USA 2012;109:12734-12739.

123 Levashina EA, Moita LF, Blandin S, Vriend G, Lagueux M, Kafatos FC: Conserved role of a complement-like protein in phagocytosis revealed by dsRNA knockout in cultured cells of the mosquito, Anopheles gambiae. Cell 2001;104:709-718.

124 Warr E, Das S, Dong Y, Dimopoulos G: The Gram-negative bacteria-binding protein gene family: its role in the innate immune system of Anopheles gambiae and in antiplasmodium defence. Insect Mol Biol 2008; 17:39-51.

125 Garver LS, Xi Z, Dimopoulos G: Immunoglobulin superfamily members play an important role in the mosquito immune system. Dev Comp Immunol 2008;32:519-531.

126 Kajla MK, Andreeva O, Gilbreath TM 3rd, Paskewitz SM: Characterization of expression, activity and role in antibacterial immunity of Anopheles gambiae lysozyme c-1. Comp Biochem Physiol B Biochem Mol Biol 2010;155:201-209.

127 Kajla MK, Shi L, Li B, Luckhart S, Li J, Paskewitz SM: A new role for an old antimicrobial: Lysozyme c-1 can function to protect malaria parasites in Anopheles mosquitoes. PLoS One 2011;6:e19649.

128 Lapcharoen P, Komalamisra N, Rongsriyam Y, Wangsuphachart V, Dekumyoy P, Prachumsri J, Kajla MK, Paskewitz SM: Investigations on the role of a lysozyme from the malaria vector Anopheles dirus during malaria parasite development. Dev Comp Immunol 2012;36:104-111.
Anopheles Innate Immune System in the Defense against Malaria Infection
J Innate Immun 2014;6:169-181 DOI: $10.1159 / 000353602$ 